\title{
Exploration on Emergency Online Teaching Mode of Russian Major
}

\author{
Xuhong He, Huan Yang \\ Department of Russian, Foreign Languages Institute, Xinjiang University, Urumqi, China \\ Email: rustime@sohu.com
}

How to cite this paper: He, X.H. and Yang, H. (2021) Exploration on Emergency Online Teaching Mode of Russian Major. Open Access Library Journal, 8: e7819. https://doi.org/10.4236/oalib.1107819

Received: August 2, 2021

Accepted: October 12, 2021

Published: October 25, 2021

Copyright $\odot 2021$ by author(s) and Open Access Library Inc.

This work is licensed under the Creative Commons Attribution International License (CC BY 4.0).

http://creativecommons.org/licenses/by/4.0/

\section{(c) (i) Open Access}

\begin{abstract}
In 2020, under the influence of the COVID-19 outbreak, primary and secondary schools have conducted emergency online teaching. This paper analyzes the online teaching of Russian majors in a western university by means of literature combing and questionnaire survey, lists theoretical problems in emergency online teaching and discusses the solutions, aiming to promote the reform of the online and offline mixed teaching mode of Russian majors in Chinese universities.
\end{abstract}

\section{Subject Areas}

Linguistics, Language Education

\section{Keywords}

Emergency Online Teaching, Russian Teaching, Mixed Teaching Mode Reform

\section{1. 引言}

2020 年春季学期新冠疫情席卷全国, 春节过后, 广大学生如何开学一时 成为社会广泛关注的一个问题。2020 年 1 月 29 日, 教育部有关负责人在接受 采访时表示 “停课不停教、不停学既是战疫情应急之举, 也是 “互联网+” 教 育的重要成果应用展示, 任务艰巨, 使命光荣。”1

中国高等教育通过实施全面的在线教学, 成功应对危机、化危为机, 实 现了延期开学、如期开课, 打了一场漂亮的阻击战。中国各高校积极讨论突 发事件的应对措施, 通过调研和培训的方式推进大规模的在线教学确保疫情 条件下教学工作正常开展, 此外前期中国国内慕课建设的积极成果和信息化 技术的培训和储备也为顺利开展在线教学提供了可能。混合式教学因其整合 了传统教学与网络教学的优点, 纷纷被各大高校所采用。

http://www.moe.gov.cn/jyb_xwfb/gzdt_gzdt/55987/202001/t20200129_416975.html. 
全疆大中小学校纷纷响应教育部 “停课不停学” 的号召, 纷纷探索在线 教学的解决方案。据统计 ${ }^{2}$, 截至 2021 年 4 月初, 全国在线开学的普通高校 共计 1454 所, 95 万余名教师开设 94.2 万门、 713.3 万门次在线课程, 参加在 线课程学习的学生达 11.8 亿人次。今年第一季度, 我国在线课程平台上线慕 课增长了 5000 门, 慕课总数已达 2.3 万门; 其他在线课程增长了 1.8 万门。

穆肃和王雅楠(2020)将紧急情况下利用互联网开展教学活动的教学形式 称为 “应急远程教学” , 在中国的实践语境中则可称之为 “应急上线教学” ([1]: p. 22)。焦建利、周晓清和陈泽璇将网络在线课程定义为: “将线下的教 学内容及教学过程数字化, 并以学习管理系统为载体, 进行基于课程内容的 线上教与学过程” ([2]: p. 106)。我们根据此次疫情影响下的应急模式的教学 特点, 将 2020 年春季学期以来进行的在线直播教学称之为 “应急在线教学” 模式, 通过这种 “紧急模式下的教学模式的改革, 能够保证在疫情的前提下 最大限度地给学习者提供学习条件和保障, 全国各个院校利用能够利用的条 件, 尤其是网络资源, 让学生获取尽可能多的知识” ([3]: p. 219)。

\section{2. 疫情时期的在线教学准备}

全国各高校都在积极探索应对疫情时期给带来的挑战, 积极学习和探索 一系列线上教学解决方案, 制定应急远程授课课程大纲, 组织教学。以中国 新疆维吾尔自治区某高校为例, 从 2020 年 1 月底就已经开始开展在线教学的 准备工作, 针对教师和学生进行的在线信息化技术与设备情况进行调查, 了 解师生进行线上教学的可行性, 同时进行教育信息化培训工作。从在线教学 平台、资源建设、教学模式、教学能力、学生监管等角度为疫情期间在线教 学提供改进建议。

我们调研了中国知网有关 “在线教学” 的文献共 1447 篇核心期刊论文 ${ }^{3}$, 发文可视化分析如图 1 所示。我们发现, 高校教师群体中普遍存在的信息化

\section{总体趋势分析}

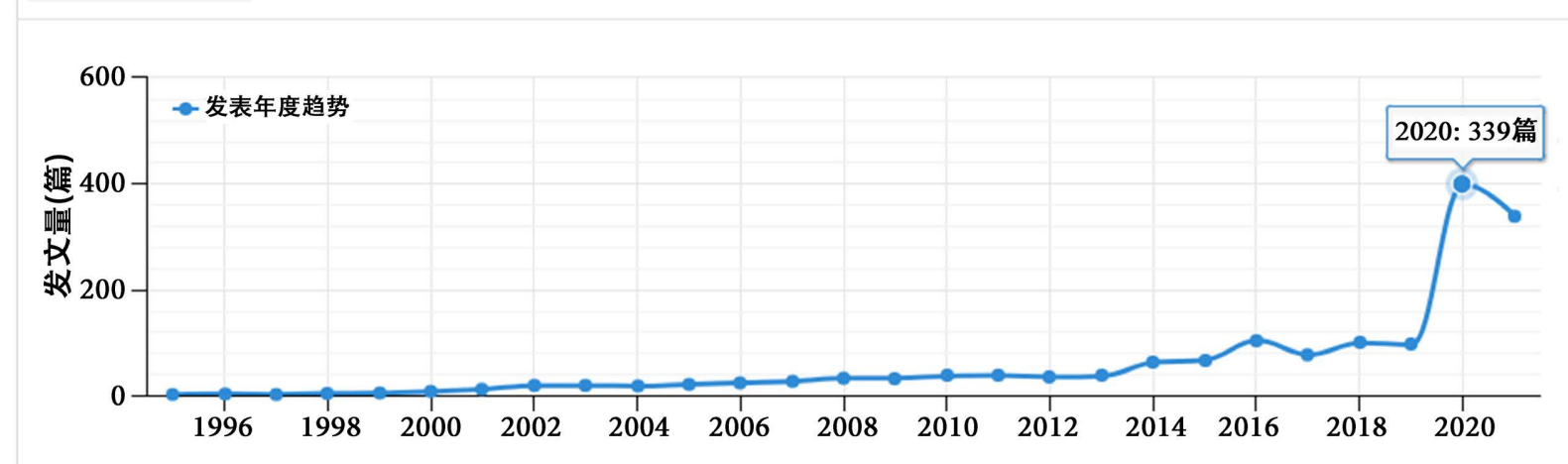

图 1. “在线教学” 主题发文量总体趋势分析

${ }^{2}$ https://news.tsinghua.edu.cn/info/1003/78348.htm。

3数据来源: https://kns.cnki.net/KNS8/Visual/Center, 检索日期 2021 年 8 月 10 日。

文献总数: 1447 篇; 检索条件: (主题\% = “在线教学” or 题名 $\%=$ “在线教学” or title = xls (“在 线教学') or v_subject = xls (“在线教学')) AND ((来源标识码 = 'P01') OR (来源标识码 = 'P0209')); 检索范围: 期刊。 
教学能力不足、在线教学的心理准备不够([4]: p. 57); 教学平台不够完善(张 玲 2020) [5], 在线教学的课程设计经验不足, 注重形式, 忽略学习内容的编 排、忽视教学设计例如何结合在线教学有针对性的对教学内容进行选择、对 教学媒介选择、课程讲解思路、活动设计等问题。

在线课程直播教学模式在满足应急在线教学的同时, 仍然存在一定的问 题, 包括网络授课和学习环境以及硬件支持不足、课题互动过程中交互性性 弱等特点 [6]。同时, 在整个在线课程教学过程中, 教师不了解学生的具体学 习效果, 对于确定下一个教学计划缺乏可靠的依据。提出一种实时交互技术 以增强整个在线课程教学平台的互动性; 结合学习过程的监控方法, 在整个 在线课程学习过程中展示学生的学习效果, 最终构建在线课程直播教学平台。 测试结果表明, 在线课程直播教学平台可以提高学生的教学效果。

对于语言类专业课程而言, 在线教学的模式并未得到广大教师的认可, 以本文调研的高校为例 ${ }^{4}, 2020$ 春季学期进行了直播教学调查, 在 835 名参 与调查的教师中, 583 人 $(69.82 \%)$ 认为自己所教授的课程适宜采用线上教学的 形式适宜，105 人(12.57\%)选择不适宜，147 人(17.60\%)不确定是否适宜线上 教学模式(图 2), 有超过半数(547, 占 $65.5 \%$ )的老师认为自己掌握了大部分学 生的学习情况(图 3)。

超过半数的教师 (51.61\%)认为学生学习状态良好, 有六成以上的教师 (62.51\%)希望获得培养学生自主学习能力方面的指导。如何调动学生学习兴 趣、如何开展有效的师生互动、在线教学的课程设计方法也分别是教师们希 望获得的主要培训内容。参与调查的 100 名语言类课程(含大学英语通识必修 课和语言类专业课)教师之中, 72 人认为自己的课程适宜线上直播教学模式, 12 人认为所教课程不适宜在线直播教学, 16 选择人不确定是否适宜。

通过针对该校俄文全体学生发布的《俄语语法》在线教学问卷调查结果 显示 ${ }^{5}, 80.75 \%$ 的学生认为《语法》课程在专业学习中非常重要; $61.5 \%$ 的学

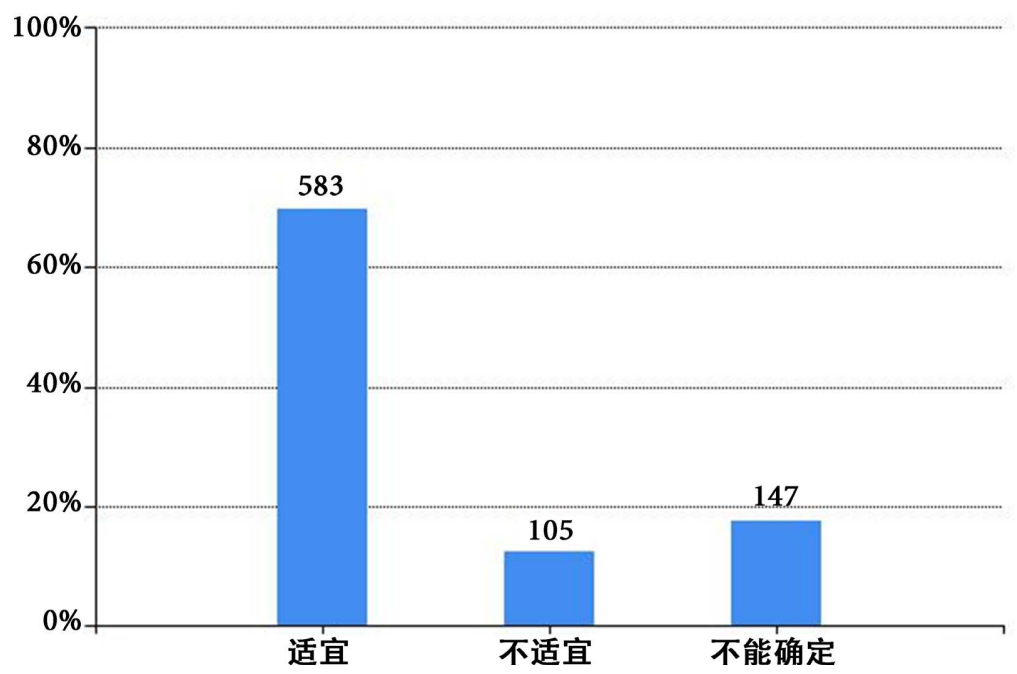

图 2. 教师对在线教学授课形式的评价

4 图 2 和图 3 的部分数据参考本校教务处的 2020 年春季学期调研的公开数据, 在此表示感谢。 5 该问卷由本系俄语语法课程教学团队教师编制, 使用问卷星平台进行发布, 共收到有效问卷 187 份。 


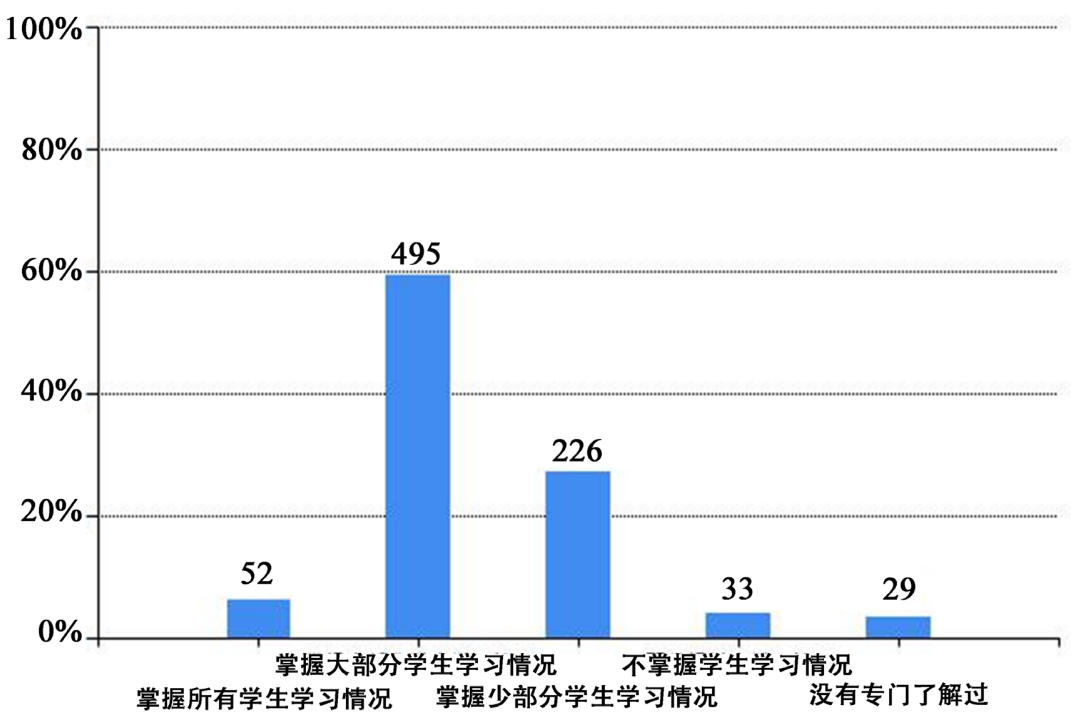

图 3. 教师对在线课堂学习情况的掌控情况

生认为《语法》课程对其他课程学习有积极的影响; 85.03\%的学生表示对《语 法》课程有兴趣。结果说明《语法》课程在专业学习中占据着十分重要的地 位, 学生对该课程也保持着极高的重视程度与兴趣。94.66\%的学生表示对《语 法》课程授课情况满意。9 $6.8 \%$ 的学生表示对《语法》课程教授过程中所使用 雨课件等教学工具表示满意, $3.2 \%$ 的学生针对雨课件的设计与使用提出了相 应建议, 表示有时课件切屏过慢, 影响课堂学习, 更偏向于在课堂上有老师 直接交流。

\section{3. 在线教学工具的使用}

经过该地区部分高校的俄语专业教师一方面参与学校组织的教学软件与 平台的培训, 另一方面自己也在积极探索各类在线会议系统软件如 “钉钉” 、 “腾讯会议”、腾讯课堂、雨课堂平台、CCTALK、zoom、通讯软件腾讯 $\mathrm{QQ}$ 、微信等, 以此来实现信息化技能提升。通过开学前超过半个月密集的软 件培训工作, 到 2020 年 2 月 16 日, 该校俄语专业的课程几乎全部采用直播 教学的方式, 主要的授课形式是利用专业的教学管理软件平台学堂在线 (Xuetang.X)提供的基于微信的插件 “雨课堂” , 在 PowerPoint 软件中实现运 行。雨课堂授课为学生提供了最便捷的在线学习方式, 只要一部智能手机和 一个微信账号就可以在线听课。

2020 年春季学期, 该校俄语专业共开设 22 门专业课程, 其中 18 门为实 践类课程, 授课教师共 21 人。参与学习的学生达到 350 余人。通过半个多月 的努力, 俄语专业在开学时直播授课的课程比例达到 $90 \%$ 以上。

然而对于语言类专业, 尤其是俄语专业来说, 在线直播教学的互动性弱; 缺乏可用慕课资源、SPOC 建设数量和质量不能满足教学需要; 如何实现实 质等效的要求, 以及慕课教学管理经验不足等问题也是教师和教学管理人员 关注的问题。

在线教学准备阶段, 存在着俄语在线教学平台资源建设数量少, 课程不 
足等问题, 可用的俄语慕课资源有: 智慧树 (http://www.zhihuishu.com)、中 国高校外语慕课联盟(http://www.moocs.unipus.cn)、爱课程 (http://www.icourses.cn)、腾讯课堂(https://ke.qq.com)、沪江网 (https://www.hujiang.com)等。

在线运行课程的时候需要关注很多因素: 例如学生在线学习的进度、讨 论社区的主题讨论维护、对学生的问题进行选择性回复, 组织有效的在线讨 论、通过微信群 QQ 群等方式与学生进行交流, 促进课堂教学的互动、及时 更新教学内容; 每周都应有 “形成性测试” 保证课堂教学质量; 针对学生的 线上教学内容需要有效组织线上直播。

目前有很多数字化教学工具为混合式教学的利器被广泛使用, 利用雨课 堂的教学平台能够连接师生的智能终端, 将 “课前 - 课上 - 课后” 的每一个 环节都赋予全新的体验, 最大限度地释放教与学的能量, 推动教学改革。

雨课堂将复杂的信息技术手段融入到 PowerPoint 软件和微信之中, 在课 外预习与课堂教学间建立沟通桥梁, 让课堂互动永不下线。使用雨课堂, 教 师可以将带有 MOOC 视频、习题、语音的课前预习课件推送到学生手机, 师 生沟通及时反馈; 课堂上实时答题、弹幕互动, 为传统课堂教学师生互动提 供了完美解决方案。雨课堂科学地覆盖了课前 - 课上 - 课后的每一个教学环 节, 为师生提供完整立体的数据支持个性化报表、自动任务提醒, 让教与学 更明了。雨课堂还不断进行更新和升级, 在 2 月底 3 月初经历了巨大的网络 流量压力考验, 据学堂在线雨课堂负责人介绍, 2020 年 3 月使用雨课堂的师 生达到 3100 多万人，有 2600 多所高校同时使用，雨课堂在疫情期间不断扩 容, 并且上线了荷塘、长江、黄河雨课堂, 等服务器, 2020 年 4 月 21 日开始 推出国际版, 能够满足不同国家学习群体的使用需求。

使用雨课堂授课能够保证整个教学环节的完整性, “课前 - 课中 - 课后” 三段式推进在线教学质量提升。雨课堂提供课前预习材料的推送, 学生会及 时在微信公众号和小程序收到消息提醒, 预习课件每页都可以插入语音, 单 条语音最长 60 秒, 条数不限。云空间存储教学材料, 方便快捷, 利于学生巩 固和复习。开启雨课堂授课之后, 雨课件的材料会直接上传到云端, 结束授 课以后会自动记录直播课堂的授课情况, 便于学生多次回放, 加深理解。学 生可以导出及打印教师的课件。雨课堂可以记录学生的学生行为、形成过程 性评价。雨课堂能够完整的记录学生停留在每一张 PPT 上的时间, 自动生成 每个学生个性化的学习报告, 并根据课堂表现提供优秀学生名单和预警学生 名单; 评价较为公正客观; 即时提供学情反馈, 雨课件有插入题目的功能, 题型有选择题、投票题, 填空题、主观题等类型, 学生通过投稿的形式完成 课堂练习。在规定时间内完成题目后, 教师端会可以查看学生答题情况, 便 于教师直接准确的掌握该知识点学生的理解程度。在课程直播的过程中产生 的流量少, 程序运行不占用手机内存。制作好的雨课件都可上传至云端, 学 生只要进入雨课堂的公众号就可以轻易获取上课的课件。同时, 使用雨课堂 授课也便于教学管理, 每门课程可以设置协同教师, 协同教师可以让多位教 师一起教授或管理同一班级课程, 进行答疑、讨论等活动, 雨课堂还推出了 
网页版的测试功能, 便于教学单位组织测试、形成过程性评和全方位的最终 评价。

\section{4. 问题与反思}

对于俄语专业而言, 在线教学的互动与线下课堂存在差距, 对于语言类 的学习更有困难, 雨课堂的使用虽然解决了很多技术问题, 但是对于高频次 的俄语课堂互动, 显得有些薄弱, 学生只能通过弹幕进行文字的反馈, 无法 实时与教师进行语音或视频的交互。我们通过不断的摸索, 形成了自己的解 决的方案, 开启 “腾云驾雨” ${ }^{6}$ 模式, 即使用腾讯会议开摄像头、进行直接对 话+雨课堂进行课前课后的课件发送、课堂展示、录制屏幕、发送练习题目以 及测试等多功能立体的教学手段和模式; 为保证学习效果, 帮助学生建立学 习小组, 通过 $\mathrm{QQ}$ 群、微信群的群聊功能和文件传输的功能, 督促学生进行 打卡学习, 互相监督、同时进步。教师要经常在小组内进行答疑, 让学生感 受到老师对他们的关注。形成激励机制, 能够看到每个小组、每个人的进步, 组员分配要合理, 鼓励组长提高组织能力。让学生在你的课堂上有获得感而 不是挫败感。针对在线测验和考试的环节我们也进行了探索和试验, 较为客 观反映学生学习的考核方式是增加过程性评价的比例, 将平时成绩由 $30 \%$ 提 升至 50\% 60\%, 利用雨课堂网页版编制在线试题, 在考试的时候进行双机位 监考, 确保考试的公平公正。

对俄语专业来说, 教学内容多, 又没有太多的资源可以利用, 通过网络 授课的方式保证教学质量的实质等效, 是全校师生面对的巨大困难和挑战。 我们不断探索如何让学生产生内部驱动力, 在没有老师监督的时候也能够主 动学习。

\section{1. 关注学生认知规律}

让教学设计能够符合网络环境下的认知规律, 课堂上, 要积极使用各种 方式保持学生的专注力, 课后让学生能够主动、高效的学习。在线授课的模 式下, 从教师系统讲授为唯一方式, 到学生自主、合作的学习探究的转变, 关注学生学习过程中问题意识和创造性思维品质的培养, 无论是对已往以知 识学习为中心的 “目标 - 策略 - 评价” , 还是以经验习得为中心的 “活动 体验 - 表现”, 这无疑都是一个重要突破。

充分利用分组学习模式, 让教学设计能够符合网络环境下的认知规律, 课堂上, 保持学生的专注力, 课后让学生能够主动、高效的学习。

以俄语语法课程为例, 本课程是俄语专业学生基础阶段核心课程, 每周 4 学时, 网络直播教学 8 周, 总计 32 学时。对于零起点学生来说, 周学时只 有 4 小时, 并且没有合适的远程教学资源, 《俄语语法》的课程只能靠教师 直播讲授, 学生的自主学习能力和思维水平也有待提高。通过建设在线慕课 课程资源, 学生能够在课前进行个性化的主动学习, 在课堂活动中表现更为 高效和积极, 在学习实践过程中更能锻炼自己的高阶思维能力。

6 “腾云驾雨” 的表述是新疆大学外国语学院王彩霞老师在 “一路同行” 教师工作坊的讲座中首 次提出, 在此引用并表示感谢。 


\section{2. 做好资源建设、促进学生高效主动学习}

教学平台资源是当前影响线上教学质量的主要因素。部分平台的功能还 不够全, 需要结合多平台完成一门课程教学, 特别是在师生互动功能方面不 够完善, 在测验、作业等设置上较为单一([5]: p. 192)。因此, 做好平台建设 对于线上及混合式教学至关重要。对于俄语专业而言, 首先要加快俄语语法 课程资源, 加强线上及混合式教学研究, 探索线上融合式教学新模式, 开展 行动研究; 加强教师业务培训及信息化能力培训, 从三个层面提高信息化教 学能力和水平: 会用基础的信息教育教学技术, 完成 “停课不停学” 的在线 教学任务; 用好信息教学技术, 优化技术, 利用教学组织、呈现方式来解决 师生学习和情感参与不足的问题; 驾驭信息化教学技术, 提高师生教学效能, 培养学生自主、高效、专注的学习习惯, 培养学生规划协调的工作能力。

教师要不断钻研教学内容, 向学生提出富有挑战度、促进理解、提升高 阶思维能力的问题, 追求把外在的知识内容转化为学生内在的精神力量, 创 新教育教学设计, 促进师生知能与精神的成长, 要实现从培训干预到自然形 成再到自觉成长转变([6]: p. 54)。李妍(2020)也通过实践结果证明, “通过智 慧教学的课程模式与评价, 师生互动反馈明显增强, 学生学习的积极性显著 提升了, 同时互动反馈大数据有望实现多方位评价学生能力的模式。” ([7]: p. 97)。师生互动反馈更加实时、高效、智能。网络在线平台构建了课前、课上、 课下多元互动反馈教学模式, 师生共同反思课堂, 并提出客观的教学评价方 法。

\section{3. 优化评价模式体系}

多元化教学评价, 过程性评价与终结性评价相结合。在平时成绩评定中, 关注学生能力的培养, 关注学生能力和品格提升。以俄语语法课程为例, 考 核重点主要考查俄语语法的基本概念、基本理论和基本知识, 测评学生的理 解、判断、分析、综合等能力。通过学习本课程, 在讲解语法理论的同时, 通 过大量的具体例句来培养学生的实际运用能力及口语表达能力。线下课堂的 练习情况很容易直观进行评价, 而远程线上教学过程的检测困难, 造成了学 习效果的评价与评估的复杂, 这也是影响在线教学效果的重要因素。根据学 生远程学习的方式和特点, 我们尝试将线上远程教学实施期间考核成绩模式 进行调整, 将考勤成绩设定为平时成绩的 $10 \%$, 加大了过程性评价的占比, 作业占平时成绩的 $30 \%$, 网上讨论等学习活动占平时成绩的 $10 \%$, 在线测验 占 $50 \%$ 。在综合成绩中, 平时成绩与期中测验成绩即过程性评价成绩占比 $40 \% \sim 60 \%$, 期末笔试成绩占 $40 \%$ 、口试占 30\%。在主要考查基本概念和基本 理论的同时, 兼顾测评学生的理解、判断、分析、综合等能力和思想政治表 现。最终考核成绩中降低期末考试卷面成绩在评价体系中的比例, 增加学生 课堂参与度的过程性评价比重。借助教学研究成果, 优化评价体系, 设计 “小 组作业微评价量表” 使学生能够产生主动学习的动力, 培养学生的自信心, 更有利于知识的重构, “这种评价方法量化测评混合学习效果, 全面评估学 习行为” ([8]: p. 107)。 


\section{5. 结语}

随着后疫情时代来临, 我们在线教学管理过程中从忙乱无措到游刃有余, 总结出做好顶层设计、建立在线教学专家咨询团队、预设出备选方案、进行 应用数据分析、培训教学督导、教师相互听课等多种途径进行在线教学的组 织和监督的管理方法。疫情期间在线教学应充分利用线上教学平台和资源, 鼓励学生利用个性化学习的手段; 成立在线课程评估小组, 科学建设在线课 程、建立在线教学质量保障机制, 促进在线教学规范化、建立在线课程学生 考评机制, 加强对学生的引导与监督, 个性化评价, 建立学生档案, 帮助学 生建立合适的学习小组, 展开合作学习。

通过这一年以来的在线教学 “练兵”, 我们积累了在线教学经验, 建立 了俄语语法课程的慕课资源，并探索出一套适用于西部高校俄语专业线上线 下混合的教学模式, 改变了教师的教学形式、学生的学习方式、优化了人才 培养的管理模式, 融合了 “互联网+” 和信息技术, 推进教育教学改革, 促进 本专业人才培养质量的稳步提升。后疫情时代的在线教学该如何进行, 俄语 专业的慕课资源的贵乏，对慕课学员缺少实时或个性化的支持，学习支持服 务谁来做？怎么做？导学、督学、助学的任务和效果如何完成等都是未来一 段时间我们要思考的问题。

\section{基金项目}

本文受 2018 年新疆维吾尔自治区教学改革项目 “基于 SPOC 平台的《俄 语语法》慕课建设与翻转课堂研究” (项目编号：2018JG21)资助。

\section{Conflicts of Interest}

The authors declare no conflicts of interest.

\section{References}

[1] 穆肃, 王雅楠. 转“危”为“机”: 应急上线教学如何走向系统在线教学 [J]. 现代远 程教育研究, 2020, 32(3): 22-29.

[2] 焦建利, 周晓清, 陈泽璇. 疫情防控背景下 “停课不停学”在线教学案例研究 [J]. 中国电化教育, 2020(3): 106-113.

[3] 张亚宁. 2020 年新冠肺炎疫情下学生停课不停学的分析与探索[J]. 汽车实用技 术, 2020, 45(20): 219-220.

[4] 杨淑莲, 刘兰娟. “停课不停教、停课不停学”背景下高校在线教学实践研究一一 以上海财经大学为例 [J]. 中国教育信息化, 2020(23): 59-63.

[5] 张玲. 疫情防控背景下高校“停课不停学”在线教学实践探索 [J]. 大学教育, 2020(9): 190-193.

[6] 赵晓伟, 沈书生. 在线教育中教师 TPACK 塔式认知层次构建策略[J]. 现代远距 离教育, 2019(3): 49-55.

[7] 李妍. 智慧教学的课程模式与评价[J]. 大学教育, 2020(11): 97-100.

[8] 邓晓宇, 张品. 基于 SPOC 双线混融教学促进深度学习的行动研究[J]. 教育学术 月刊, 2020(11): 106-111. 


\section{Appendix (Abstract and Keywords in Chinese) 应急在线俄语专业教学模式探索}

摘要: 2020 年在新冠肺炎疫情影响下, 全中国大中小学校师生都进行了一场 应急模式在线教学实践，本文通过文献梳理及问卷调查等方式分析了西部某 高校的俄语专业在线教学情况, 列举应急在线教学中的理论问题并讨论解决 方案，旨在推动我国高校俄语专业线上线下混合式教学模式改革。

关键词：应急在线教学，俄语教学，混合式教学模式改革 